\title{
Interessant, vielseitig - und immer rasch ausgebucht
}

\section{Pädiatrischer Fortbildungskurs Obergurgl}

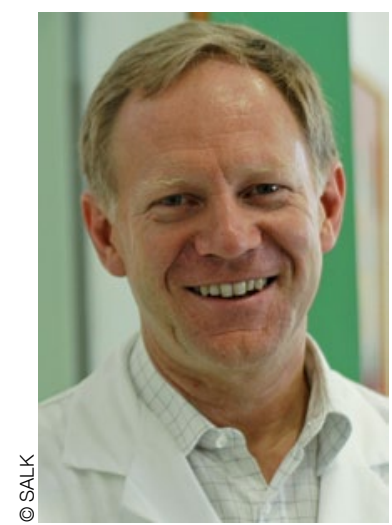

Univ.-Prof. Dr. Wolfgang Sperl Vorstand der Univ.-Klinik für Kinder- und Jugendheikunde, SALK, und Leiter der oben genannten Fortbildungsveranstaltung

Pädiatrie \& Pädologie: Ihre Fortbildungsveranstaltung ist zur Institution geworden. Ist das für Sie ein Auftrag?

SPERL: Gute Fortbildung in der Kinderheilkunde dauerhaft zu organisieren ist tatsächlich ein Auftrag. Ich verstehe darunter besonders, dass Einbeziehungen aller Subspezialitäten der Pädiatrie und auch der Randgebiete bzw. der Schnittstellen hin zur Kinder- und Jugendpsychiatrie bzw. Kinderchirurgie. Weiters ist die Fortbildung in Obergurgl länderübergreifend, d.h. horizontüberschreitend gemeint, weil wir immer wieder Referenten aus den D-A-CH Ländern einladen und wir in der deutschsprachigen Pädiatrie voneinander profitieren können.

\section{Es ist gerade das wissen- schaftlich Neue und prak- tisch relevante Anwendbare, das wir hier immer wieder vorstellen wollen.}

\section{Gibt es ein Hauptthema?}

SPERL: Nein, bewusst werden jedes Jahr alle Themen der Pädiatrie jeweils einbezogen. Es gibt ein breit aufgestelltes Programmkomitee, das praxisrelevante Vorschläge einbringt. Eine Konstante ist immer die kinderradiologische Fortbildung am
Dienstagnachmittag, aber auch der Bericht vom wissenschaftlichen Vorsymposium am Montagnachmittag, das meistens Ernährungs-, Impf- und immunologische Fragen behandelt.

Welche Themen behandelt der Fortbildungskurs 2013, möchten Sie einige besonders hervorheben?

SPERL: Der Fortbildungskurs 2013 behandelt verschiedene Themen wie Infektiologie, Neonatologie, Psychosomatik, Kinderorthopädie und pädiatrische Gastroenterologie, Augen- und Ohrenerkrankungen. Besonders hervorheben möchte ich die erstmalige Sitzung „Klinik-Hautnah“, hier werden Fälle präsentiert für Blickdiagnosen-problemorientiertes Lernen, und „Lernen aus Fehlern“. Ich erwarte Interaktivität, einen guten Einbezug des Voting-Systems und viele Engramme, die mit nach Hause genommen werden.

\section{Was macht die Attraktivität des Kurses aus?}

SPERL: Es ist die geniale Kombination von sehr guter Fortbildung, dem länderübergreifenden Treffen aller Kinder- und Jugendfachärzte aus den verschiedenen Bereichen, Universitätskliniken, Krankenhäuser, aber auch aus der Praxis, das Treffen von jung und alt und die besondere Kombination zwischen der Fortbildung, Freundschaftspflege und der Freitzeitmöglichkeiten in der schönen Natur.

Fortbildung: Praxis und Wissenschaft oder schließt das eine das andere aus?

SPERL: Nein, es ist gerade das wissenschaftlich Neue und praktisch relevante Anwendbare, das wir hier immer wieder vorstellen wollen. Daher sind Experten der jeweiligen Subspezialitäten eingeladen, die Teilnehmer upzudaten und Wissenschaft in die Praxis zu übersetzen.

\section{Ist der Veranstaltungsort zeitgemäß}

SPERL: Obergurgl ist eine Marke geworden. Wir würden den Veranstaltungsort nicht gerne ändern. Wir sind beliebte Gäste in einer Zeit der Zwischensaison. Die Jahreszeit und der Ort sind für die Fortbildung ideal geeignet.

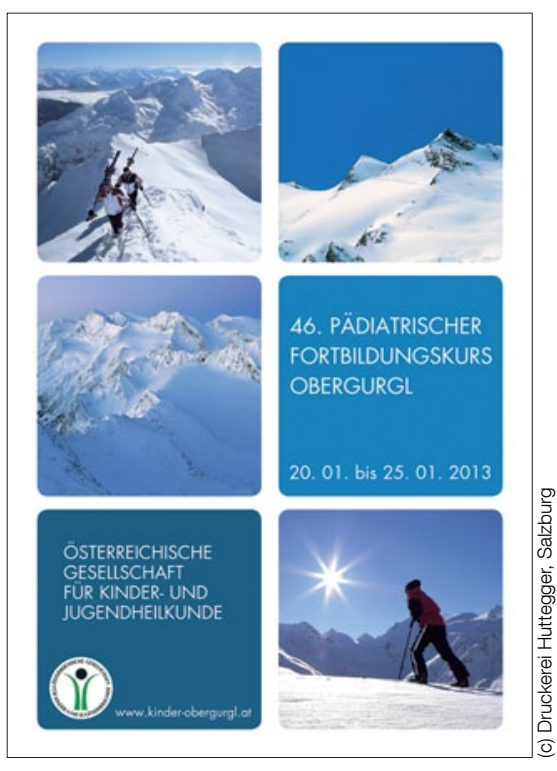

Haben Sie überlegt, den Kurs abzuändern und wenn ja, in welche Richtung? SPERL: Der Andrang ist sehr groß, wird sind innerhalb weniger Tage immer wieder ausgebucht. Glücklicherweise wird der Piccard-Saal in 2 Jahren ausgebaut, da werden wir endlich mehr Teilnehmer aufnehmen können, dzt. sind wir mit 300 limitiert.

Was wünschen Sie sich für den Kurs 2013?

SPERL: Den Teilnehmern und mir eine unfallfreie Woche, viel, was für die Praxis und für das Arbeiten mit den Kindern und Jugendlichen mit nachhause genommen werden kann, Freundschaften pflegen, sich auf hohem Niveau auch zwischenzeitlich vom Klinikalltag entspannen.

Wird es 2014 wieder einen pädiatrischen Fortbildungskurs Obergurgl geben? SPERL: Am Mittwoch der Fortbildungswoche sitzt das Programmkomitee zusammen und erstellt das Programm für 2014. Ich hoffe, dass es wieder abwechslungs- und inhaltsreich wird und zahlreiche Kinderärztinnen und -ärzte nach Obergurgl lockt.

Herzlichen Dank!

Dr. R. Höhl 